\title{
Erratum
}

\section{Erratum to: High-efficiency approach for fabricating MTE rotor by micro-EDM and microextrusion}

GENG Xuesong ${ }^{1}$, CHI Guanxin ${ }^{1}$, WANG Yukui ${ }^{1}$, and WANG Zhenlong ${ }^{1,2, *}$

1 School of Mechanical Engineering, Harbin Institute of Technology, Harbin 150001, China

2 Key Laboratory of Micro-systems and Micro-structures Manufacturing, Ministry of Education, Harbin Institute of Technology Harbin 150001 China

Erratum to: Chinese Journal of Mechanical Engineering

DOI: 10.3901/CJME.2014.0505.086

The original version of this article unfortunately contained a mistake. The presentation of the DOI in printed version was incorrect. The corrected DOI is given below.

DOI: 10.3901/CJME.2014.0527.101

* Corresponding author. E-mail: wangzl@hit.edu.cn

Supported by National Basic Research Program of China (973 Program, Grant No. 2012CB934102), National Natural Science Foundation of China (Grant No. 50835002), and National Science Foundation for Young Scientists of China (Grant No. 51105111)

(C) Chinese Mechanical Engineering Society and Springer-Verlag Berlin Heidelberg 2014 